\title{
Estratégias de coping durante a vivência do câncer de mama
}

\author{
Coping strategies during the experience of breast cancer \\ Estrategias de enfrentamiento durante la experiencia del cáncer de mama
}

Tayanne Queiroz Porcinio Cirqueira ORCID: https://orcid.org/0000-0002-1470-8355 Universidade Federal do Maranhão, Brasil E-mail: tayanne.queiroz@hotmail.com

Adriana Gomes Nogueira Ferreira ORCID: https://orcid.org/0000-0001-5185-2092 Universidade Federal do Maranhão, Brasil E-mail: adriana.nogueira@ufma.br

Cícera Rodrigues de Sousa Cunha ORCID: https://orcid.org/0000-0003-3458-9151

Universidade Federal do Maranhão, Brasil E-mail: cicera.cunhaenf@hotmail.com

Francisco Dimitre Rodrigo Pereira Santos ORCID: https://orcid.org/0000-0003-3036-7631 Universidade Estadual do Tocantins, Brasil E-mail: franciscodimitre@hotmail.com

Ismália Cassandra Costa Maia Dias ORCID: https://orcid.org/0000-0002-9203-0869 Universidade Federal do Maranhão, Brasil E-mail: ismalia.dias@ufma.br

Janaina Miranda Bezerra

ORCID: https://orcid.org/0000-0002-4799-9638 Universidade Federal do Maranhão, Brasil E-mail: janaina.mb@ufma.br

Juliana Gomes Nogueira Ferreira ORCID: https://orcid.org/0000-0002-2041-1267 Instituto Superior de Teologia Aplicada, Brasil E-mail: julianagnf82@gmail.com

Miguel Henrique da Silva dos Santos ORCID: https://orcid.org/0000-0003-1737-2137 Universidade Federal do Ceará, Brasil

E-mail: miguelhenrique100@ hotmail.com

Aldo Lopes da Costa Júnior

ORCID: https://orcid.org/0000-0002-4216-4690

Universidade Federal do Maranhão, Brasil E-mail: jrlopesc@gmail.com

\begin{abstract}
Resumo
Com o objetivo de conhecer o coping de mulheres com diagnóstico e tratamento de câncer de mama, através dos relatos de vida, foi realizado estudo qualitativo, do tipo História Oral, em 2016, com cinco mulheres do interior do Nordeste do Brasil. A coleta se deu por meio de entrevista semiestruturadas no domicílio das participantes, após contato prévio com a instituição de saúde a qual estas eram vinculadas. As entrevistas foram gravadas, transcritas e analisadas, de acordo com a técnica de análise de conteúdo de Bardin e auxílio do software Web Qualitative Data Analysis (WebQDA). A partir dos relatos, surgiram as categorias: Atitudes de autocontrole e Apoio Social no enfrentamento do Câncer de mama, e o Coping religioso no câncer de mama. As mulheres do estudo expressaram autocontrole, suporte social e coping religioso no enfrentamento do diagnóstico e tratamento do câncer de mama. Para o planejamento de assistência de enfermagem e da equipe multiprofissional é importante conhecer o coping da mulher durante o diagnóstico e tratamento do câncer de mama.
\end{abstract}

Palavras-chave: Câncer de mama; Enfermagem; Enfrentamento.

\begin{abstract}
Aiming to know the coping of women diagnosed and treated for breast cancer, through life reports, a qualitative study, of the Oral History type, was carried out in 2016 with five women from the interior of Northeastern Brazil. Data collection took place through semi-structured interviews at the participants' homes, after prior contact with the health institution to which they were linked. The interviews were recorded, transcribed and analyzed, according to Bardin's content analysis technique and the help of the Web Qualitative Data Analysis (WebQDA) software. From the reports,
\end{abstract}


the following categories emerged: Self-Control Attitudes and Social Support in Coping with Breast Cancer, and Religious Coping in Breast Cancer. The women in the study expressed self-control, social support and religious coping in coping with the diagnosis and treatment of breast cancer. For the planning of nursing care and the multidisciplinary team, it is important to know the woman's coping during the diagnosis and treatment of breast cancer.

Keywords: Breast cancer; Nursing; Confrontation.

\section{Resumen}

Con el objetivo de conocer el afrontamiento de las mujeres diagnosticadas y tratadas por cáncer de mama, a través de relatos de vida, en 2016 se realizó un estudio cualitativo, de tipo Historia Oral, con cinco mujeres del interior del noreste de Brasil. La recolección de datos se realizó mediante entrevistas semiestructuradas en los domicilios de los participantes, previo contacto con la institución de salud a la que estaban vinculados. Las entrevistas fueron grabadas, transcritas y analizadas, de acuerdo con la técnica de análisis de contenido de Bardin y la ayuda del software Web Qualitative Data Analysis (WebQDA). De los informes surgieron las siguientes categorías: Actitudes de autocontrol y apoyo social para afrontar el cáncer de mama y afrontamiento religioso del cáncer de mama. Las mujeres del estudio expresaron autocontrol, apoyo social y afrontamiento religioso para afrontar el diagnóstico y el tratamiento del cáncer de mama. Para la planificación de los cuidados de enfermería y el equipo multidisciplinario, es importante conocer el afrontamiento de la mujer durante el diagnóstico y tratamiento del cáncer de mama.

Palabras clave: Cáncer de mama; Enfermería; Confrontación.

\section{Introdução}

O câncer é uma patologia que ocasiona inúmeras implicações na saúde do indivíduo, por esse motivo, no momento do diagnóstico positivo é primordial iniciar o tratamento, que pode ser procedimento clínico invasivo, com utilização de drogas classificadas como quimioterápicas e radioterapias (Paganine, 2016).

Dentre os diversos tipos de câncer, o que mais acomete as mulheres é o de mama. No Brasil, em 2020 foram 66.280 casos novos. Nas capitais brasileiras, esse número corresponde a 19.820 casos novos a cada ano. A taxa bruta de incidência estimada para 2020 foram 66.280 novos casos, consequentemente é a quinta causa de morte por câncer em geral no país, por representar uma taxa de incidente de 43,74 casos por 100 mulheres (Instituto Nacional De Câncer José Alencar Gomes Da Silva [BR], 2020).

Devido ao câncer de mama acometer a vida de inúmeras mulheres em todo mundo, tornou-se uma problemática de saúde pública, razão pela qual o Ministério da Saúde tem desenvolvido Políticas Públicas acerca deste assunto desde os anos 80, estimulado por um programa intitulado Viva Mulher, de 1998 (Pontes et al., 2020). Atualmente, o controle do câncer de mama é parte integrante do Plano de Ações Estratégicas para o Enfrentamento das Doenças Crônicas Não Transmissíveis (DCNT) no Brasil, 2011-2022 (Instituto Nacional De Câncer José Alencar Gomes Da Silva [BR], 2021).

O câncer independente de sua localização, causa transtorno para o indivíduo, por ser uma patologia que compromete a qualidade de vida. E tratando-se da mulher com câncer de mama, esta é uma situação que afeta também a autoestima, pois quando o diagnóstico ocorre em estágio avançado, a mastectomia é indicada, comprometendo-a física e psicologicamente. Além do mais, é sabido, que cada pessoa possui um jeito particular de enfrentar a doença (Fonseca et al., 2017; Migowski et al., 2018).

O feedback de adaptação diante do fator estressor denominado coping faz referência às estratégias cognitivas e comportamentais utilizadas por cada pessoa, para lidar com situações que requeiram a ativação da resposta ao estresse (Dias, 2019). Assim, as formas de enfrentamento são diversas, podendo ser positivas e/ou negativas, e ainda, dentre elas têm-se a resolução de problemas, a reavaliação, autocontrole, suporte social, religiosidade, fuga e afastamento (Reis, Panobianco \& Gradim, 2019).

Diante do exposto, cabe aos profissionais de saúde reconhecer as formas de enfrentamento dos pacientes para proporcionar uma assistência de qualidade, bem como, reconhecer os momentos de fuga ou afastamento, para que, desta 
forma, estabeleça relação de confiança, buscando maior adesão ao tratamento e apoio para oferecer melhor qualidade na atenção à saúde, bem como nos momentos de reavaliação, autocontrole, suporte social ou religiosidade para potencializá-los. O objetivo desse estudo foi conhecer o coping de mulheres com diagnóstico e tratamento de câncer de mama, através dos seus relatos de vida.

\section{Metodologia}

Estudo descritivo, exploratório com abordagem qualitativa (Minayo, 2013; Pereira et al. 2018) utilizando o referencial metodológico da História Oral, uma vez que esse método facilita na compreensão da realidade do objeto estudado realizado em uma clínica privada, conveniado com o Sistema Único de Saúde (SUS) de referência no tratamento de câncer localizada na cidade de Imperatriz, Maranhão, em 2016. Para a coleta de dados foi realizada entrevista conduzida por questões norteadoras sobre o tema para possibilitar que os relatos expressassem as experiências no momento do diagnóstico e tratamento de câncer de mama.

Foram incluídas mulheres com diagnóstico de Câncer de Mama; idade superior a 18 anos, que tinham concluído o primeiro ano de tratamento e com capacidades mentais e/ou emocionais de participar do estudo, desta forma foram convidadas seis mulheres, uma se recusou a participar o que totalizou cinco participantes do estudo. Para a coleta de dados, inicialmente foi solicitado o contato das participantes que realizaram tratamento de Câncer de Mama no serviço de oncologia. Em seguida procedeu-se com o convite via contato telefônico para participar do estudo e caso aceite era realizado agendamento das entrevistas. Foi realizada entrevista individual, gravada em áudio, realizada na residência das participantes conforme agendamento prévio e seguindo um roteiro para que as participantes pudessem narrar sua experiência durante o diagnóstico e tratamento do câncer de mama. Ressalta-se que uma das participantes foi entrevistada em um hotel pois era a única participante que não residia no município. As entrevistas foram fundamentadas nos relatos de vida das mulheres, partindo desde o diagnóstico até a conclusão do tratamento com duração de aproximadamente 120 minutos, em apenas um encontro cada.

Os resultados foram organizados com auxílio do software de análise de textos Web Qualitative Data Analysis (WebQDA), ferramenta eficaz na exportação e organização dos dados, o que sem dúvida atende as necessidades do investigador, por arquitetar categorias interpretativas ou descritivas (Costa, Linhares \& Souza, 2012). A análise dos dados aconteceu a partir das três fases propostas por Bardin (2011): pré-análise, descrição analítica e tratamento e intepretação dos resultados. Compreende-se que, as categorias identificadas podem apresentar uma diversidade de comportamento frente ao câncer de mama e Coping religioso. Observa-se que existe o pragmático de representações e interferências gráficas/textuais do software, sobretudo, para facilitar a compreensão dos dados apresentados.

Os aspectos éticos da pesquisa foram respeitados e o estudo foi aprovado pelo CEP da Universidade Federal do Maranhão conforme protocolo $\mathrm{n}^{\mathrm{o}} 1.165 .129$ e para assegurar o anonimato a identidade das cinco mulheres participantes da investigação foram denominadas com os nomes bíblicos de mulheres: Sara, Ester, Ana, Maria e Rute. A lista de verificação COREQ foi usada para relatar os resultados (Souza et al., 2021).

\section{Resultados e Discussão}

A faixa etária das participantes correspondeu as idades entre 43 e 76 anos, se autodeclararam: parda, indígena, negra e duas brancas. No que se refere à escolaridade: três com ensino fundamental incompleto e duas ensino superior completo. Todas tiveram filhos, uma separada, duas viúvas e duas casadas, todas residem com familiares, quatro recebem benefício do governo e outra além do benefício trabalhava como cabeleireira, todas com renda familiar de um a três salários mínimos. No 
que se refere à religião: três se declararam católicas, uma evangélica e uma sem religião no entanto se denomina espiritualizada.

Três das participantes realizaram mastectomia total, quimioterapia e radioterapia, uma realizou quimioterapia e radioterapia e outra a radioterapia. O tempo de tratamento variou entre três e 23 anos. Duas concluíram o tratamento e estão em acompanhamento e as demais estavam em tratamento no momento da entrevista.

Para a compreensão do enfrentamento da mulher frente ao diagnóstico e tratamento do câncer de mama, é necessário conhecer não somente o processo de adoecimento, mas também as estratégias adotadas em cada momento. Assim, os relatos demonstram atitudes de enfrentamento que surgiram durante a doença conforme observado na Figura 1.

Figura 1: Mapa dos Códigos Árvore construído no WebQDA.

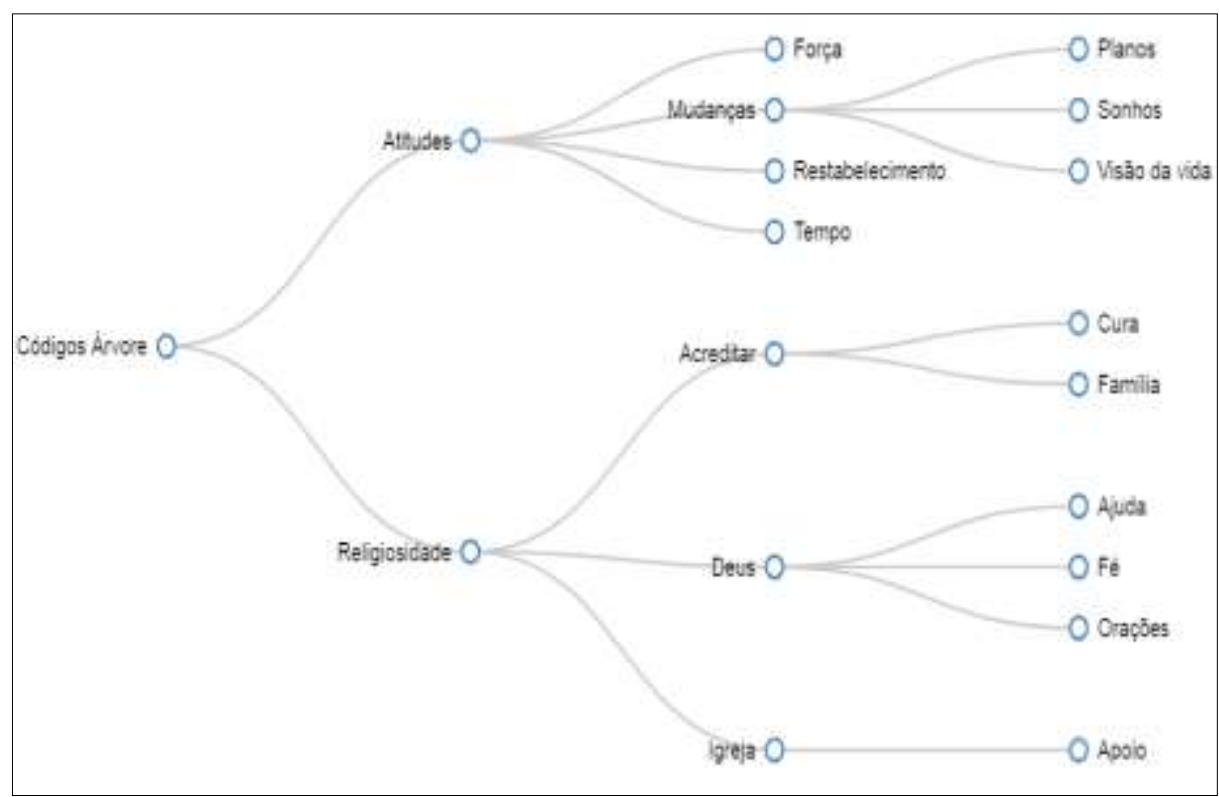

Fonte: webQDA (2016).

\section{Atitudes de autocontrole e Apoio Social no enfrentamento do Câncer de mama}

Para a mulher acometida pelo câncer de mama, é importante que desenvolva atitudes que possibilitem o enfrentamento da doença, com isso, surge a resolução de problemas, que consiste no empenho de cada uma a fim de solucionálo conforme observa-se na figura 2. A este respeito identificam-se os relatos:

“(...) Se tiver algo [sobre a doença], é tratar e vê até aonde vai (...). Desde quando descobri, já iniciei logo o tratamento, (...) para mim era tudo tirado de letra, a minha consciência sempre foi muito aberta (...)”. (Ester)

“As enfermeiras me ajudaram, me explicaram e disseram que tinha que 'tirar o peito' e eu aceitei tudo o que disseram (...), sempre digo que não estou doente, pois nunca gostei de aumentar a situação (...)”. (Ana)

“(...) Eu estava todo o tempo forte e firme e acreditando que iria ficar boa, (...) encarei o que tinha que enfrentar e fui à luta. (...) descobri uma força que não sabia que tinha. (...) Nunca fui de lamentar, nunca tinha medo, nem entrei em depressão, por que acredito que o que mata não é o câncer, mas sim a depressão." (Rute) 
Figura 2: Nuvens de palavras a partir da História Oral das mulheres portadoras de câncer de mama.

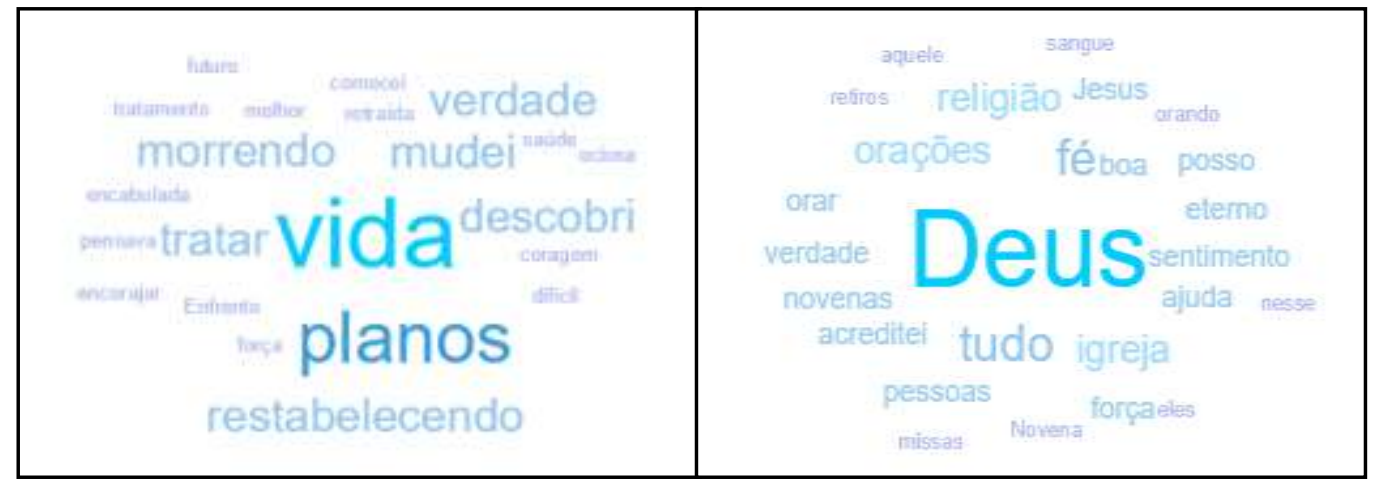

Fonte: webQDA (2016).

O autocontrole foi uma característica identificada em todas as entrevistadas, para elas, isto é fundamental para a recuperação conforme observados nos relatos a seguir:

"Na quimioterapia você não consegue comer nada, mas eu dizia, em nome de Jesus, pode ser uma casca de pau, mas eu vou comer". (...) Se você não tiver isso de pensar só positivamente, você morre.” (Sara).

“(...) Se eu fosse pensar que amanhã eu seria a próxima [a morrer] eu não estaria viva (...)”. (Maria).

Ocupar o tempo é importante no enfrentamento do câncer, visto que é uma forma de afastar pensamentos negativos e tristezas. O suporte social, que implica em desenvolver atividades sociais é uma possibilidade e faz com que as mulheres se sintam úteis, conforme evidenciado nas falas:

"Os quadros lindos, que estão nas paredes daqui de casa, fui eu quem fez. Eu fazia aquele ponto cruz, pra ver se alimentava a minha mente, para não ficar com a vida ociosa sabe, e com isso eu fui vencendo. (...) meu irmão me levava quase todo dia para tomar sorvete." (Sara)

“(...) Eu saía, trabalhava para me distrair, minha vida não mudou nesse aspecto.” (Rute).

A fuga ou afastamento que também é uma forma de enfrentamento foi identificado, não pelo fato de não aceitar o diagnóstico ou diminuir a sua gravidade como é definido, mas por manter-se retraída, querer ficar isolada, não realizar atividades que a distraíssem, como identificado no relato:

"Sempre fui uma pessoa de ficar em casa, (...). O que eu fazia era cuidar da casa, fui algumas vezes visitar a minha filha, mas fiquei mais retraída do que eu já era." (Ester).

O compartilhamento do diagnóstico positivo com pessoas próximas torna possível um melhor enfrentamento da doença, pois elas encontram conforto, apoio e sentem-se acolhidas, em especial com familiares, amigos e pessoas da igreja. A presença dessas pessoas durante o período em que as mulheres enfrentavam a doença as fortalecia para dar continuidade ao tratamento, conforme demonstrado nos relatos:

“(...) Se não fosse meu irmão eu não tinha me operado.” (Sara) 
“(...) O apoio é todo da família, (...) a fé e amizade da família, ajudam a gente a passar por isso (...). ”(Ester)

“(...) Disseram [se referindo as pessoas da igreja] que todos iam orar por mim e que eu iria ser curada e eu acreditei nisso." (Rute)

"Todo mundo me apoiou, recebi muito apoio de amigos e família, estavam todos presentes me apoiando (...) minha sobrinha que é da igreja, me acompanhou na cirurgia e em tudo, quando estava passando mal. (...) As meninas da Novena sempre estavam orando por mim, pedindo por mim e me davam força e apoio quando eu estava fraca durante a quimioterapia." (Rute)

Conforme observado nos relatos, observa-se que o apoio social foi demonstrado não somente por familiares e amigos, mas por pessoas do convívio da igreja.

\section{O Coping religioso no câncer de mama}

A religiosidade, que é caracterizada como o uso da fé para enfrentar a doença, é observada nos relatos de todas as participantes:

“(...) Eu ia à missa dia de domingo, com minha mãe, e meus irmãos. Rezo de manhã, de tarde e de noite. Se você não clamar o nome de Deus e esquecer que é o nosso único e eterno salvador e encaminhado pela nossa mãe, de que adianta? Não está fazendo nada. (...) Depois que vi Jesus sentado na minha frente, quando eu estava no coma, e nossa Senhora de Joelhos com o rosário brilhando como se fossem estrelas do céu, com as mãos postas pra Jesus e uma infinidade de mulheres com várias luzes na mão, foi aí que aquele sentimento cresceu no meu coração e passei a dizer que Deus é maior, que Deus é tudo o que temos na nossa vida (...) eu fui operada, mas foi Jesus que me curou." (Sara).

“(...) Sempre faço orações, leio a bíblia e acho que hoje faço mais, (...) acho que foi Deus que me mostrou que eu estava doente (...). Deus é o pai eterno, dono de tudo e de nós todos, tenho muita fé.” (Ester).

“(...) Vou à missa e faço orações em casa. Não tem nada impossível para Deus, pois ele pode curar tudo.” (Ana).

"A fé é uma ferramenta que ajuda qualquer indivíduo (...). O homem pode até saber a fórmula do sangue, mas ele não sabe fabricá-lo, então a gente vê Deus em tudo isso. Deus é algo inexplicável." (Maria).

"Vou à igreja, participo das novenas do mês de maio, vou às missas. Eu tinha fé que iria ficar boa. (...) Deus é a minha força e minha fortaleza. E me considero já boa.” (Rute).

De forma positiva, foi citado o aparecimento da doença como um propósito de Deus ao permitir o aparecimento da patologia, dando a esta, um significado que vai além da enfermidade:

“(...) Eu acho que se eu passo pelo que estou passando já estava determinado desde o meu nascimento (...). ” (Ester).

“(...) Eu entendia que eu tinha que passar por isso, se Deus me deu essa cruz é porque eu tinha que carregar.” (Rute).

Duas entrevistadas destacaram que a religião esteve presente em seu dia a dia durante todo o período da doença por meio de práticas e votos, conforme observado nas falas. 
"O padre colocava a mão na minha cabeça e rezava por mim e todos os nossos irmãos [...] Eu ia à missa dia de domingo, com minha mãe, e meus irmãos." (Sara)

"Durante o mês de maio inclusive, eu fiz um voto de me vestir de branco e acompanhar as novenas e na época estávamos levando a imagem de Nossa Senhora das Graças e acreditei que ela estava intercedendo por mim e por todos do grupo e no final ganhei a imagem dela." (Rute)

\section{Discussão}

A busca por tratamento, na maioria das vezes é percorrida com alguns obstáculos decorrentes do atendimento nos serviços de saúde, que é cercado por questões burocráticas, bem como pela demanda maior que a oferta de recursos disponíveis no sistema público de saúde (Almeida et al., 2015). A dor e a debilidade física em que as pacientes se encontram, demonstram a dificuldade em conviver com o problema, em que o tratamento é agressivo e doloroso (Alencar et al., 2020).

Por ser uma doença crônica, o câncer de mama merece ser entendido a partir da forma como as mulheres vivenciam a enfermidade (Machado, Soares \& Oliveira, 2017). Desta forma entender os aspectos emocionais das mulheres se torna importante, em estudo de Lacerda et al. (2020), a trajetória do tratamento do câncer de mama é marcada por incertezas quanto à cura, levando a maioria das pacientes a desacreditarem de sua identidade feminina, que pode sofrer com a perda do seio, cabelos e alterações em seu corpo. Nesse sentido, afeta diretamente seu psicológico, desencadeando sentimentos de inferioridade, incertezas e baixo autoestima (Andrade et al., 2021).

Aceitar a situação contribui para a diminuição do estresse da paciente acometida pela neoplasia, porém o espírito de luta é mais adequado a partir do momento em que se estimula a superação das dificuldades próprias do tratamento. No processo de aceitação, a mulher acometida pela doença começa a ter consciência das limitações e das possibilidades existentes (Sousa et al., 2017). A aceitação foi observada nas participantes do estudo o que demonstra uma maior possibilidade de enfrentamento no período do diagnóstico e tratamento.

De acordo com Costa et al (2019) os sujeitos empregam diversas estratégias que auxiliam a lidar com o problema de saúde de acordo com sua individualidade, partindo do que acreditam que seja eficaz. O enfrentamento do câncer de mama é um momento complexo, assim, o apoio da família e amigos auxilia na superação do medo, ansiedade e depressão, bem como no processo de aceitação do diagnóstico. Em outras palavras, o apoio social mútuo no cuidado da mulher frente ao diagnóstico da doença, serve como incentivo significativo no enfrentamento do processo de aceitação (Bittencout \& Souza, 2016) afinal, o enfrentamento do câncer de mama em alguns casos pode resultar também em consequências negativas, diferente do demonstrado pelas participantes deste estudo (Machado et al., 2017).

É importante ressaltar que a família tem um papel importante no processo de aceitação e recuperação do paciente oncológico, visto que seu acolhimento é fonte de apoio emocional, psicológico e social, e nesse vínculo afetivo, a mulher encontra forças para lidar com a enfermidade e sofrimento causado pelo tratamento, além de constituir fonte de segurança, apoio, estabilidade emocional e estimulo para lutar por sua vida (Conde et al., 2016).

Todas as mulheres do estudo demonstraram fé no enfrentamento da doença. A fé ou crença religiosa lhes propicia sentimento de paz, fazendo com que vivenciem o processo com otimismo, impulsionando-as a enfrentar as dificuldades próprias da doença e terem mais força de vontade para darem continuidade à vida (Almeida et al., 2015).

Neste sentido estudos apontam o Coping Religioso/Espiritual (CRE), que implica no uso da espiritualidade, religião e fé para enfrentar os problemas e estresses que acometem pessoas portadoras de doenças crônicas (Gonzalez et al., 2016). Estes fatores têm sido associados a vários aspectos positivos da adaptação ao diagnóstico e tratamento do câncer, reforçando sua importância na recuperação da saúde dos indivíduos afetados pela doença (Costa et al., 2019). A fé em Deus e a espiritualidade, ou CRE traz inúmeros benefícios aos pacientes acometidos por câncer, tais como, menor incidência de 
complicações durante o tratamento e aumento da longevidade (Sousa et al., 2017).

O CRE é uma forma de enfrentamento que tem como propósito diminuir o sofrimento ou obter mais esperança de cura com o tratamento, possibilitando assim, que a mulher tenha mais conforto e autoconfiança. Nesse sentido, é importante que o enfermeiro entenda os valores das crenças religiosas e espirituais do paciente, a fim de proporcionar uma assistência individualizada (Fernandes, 2016; Yoshnari et al., 2017).

Relacionado aos diagnósticos de enfermagem voltados à espiritualidade e religiosidade, a Classificação Internacional de Diagnósticos de Enfermagem (NANDA) apresenta, e é possível perceber que eles não são aplicados na assistência, pois não costumam ser identificados, ou quando são utilizados, raramente são registrados na evolução ou em suas observações (Herdan, 2018).

Reconhecer as formas de enfrentamento no câncer de mama são importantes na assistência das mulheres, pois favorece o desenvolvimento de intervenções não somente para os aspectos biológicos, mas sobretudo o psicológico e espiritual, assegurando uma atenção integral a mulher. Nessa perspectiva, é possível contribuir para uma assistência em saúde que englobe as singularidades envolvidas no adoecimento e tratamento, visando o bem-estar psicossocial e a comunicação com a equipe de saúde (Paiva et al., 2020). Nesse contexto a religiosidade se torna importante, por representar uma força que é impulsionada pela fé e pensamento positivo, de que os desafios e dificuldades existem no enfrentamento da doença, porém, podem ser superados.

Como limitação aponta-se o número pequeno da amostra e sugere-se que novas pesquisas sejam realizadas dando ênfase a este assunto, vinculando os aspectos de coping com mulheres com câncer de mama, pois quaisquer estratégias que auxiliem no enfrentamento durante o diagnóstico e tratamento, são importantes para os pacientes oncológicos, a fim de promover apoio e encorajamento durante o tratamento, podendo assim, chegar ao fim do tratamento com bons resultados, não somente físicos, mas também psicossociais e espirituais.

\section{Conclusão}

Quando a mulher é diagnosticada com câncer de mama, ela apresenta formas de enfrentamento que necessitam ser reconhecidas pela enfermagem e equipe multiprofissional, para que possam auxiliar de maneira adequada e conforme as necessidades individuais que a doença impõe. As mulheres do estudo expressaram, autocontrole, suporte social e coping religioso no enfrentamento do diagnóstico e tratamento do câncer de mama.

É importante ressaltar que autocontrole, suporte social e religiosidade apresentam influência positiva no enfrentamento da patologia. Assim a identificação das formas de enfrentamento de mulheres com câncer de mama, são importantes para o planejamento de assistência de enfermagem e da equipe multiprofissional de modo a auxiliar à mulher durante o diagnóstico e tratamento do câncer de mama.

\section{Agradecimentos}

Á Coordenação de Aperfeiçoamento de Pessoal de Nível Superior - Brasil (CAPES) - Código de Financiamento 001. À Fundação de Amparo à Pesquisa e ao Desenvolvimento Científico e Tecnológico do Maranhão (FAPEMA) pelas bolsas de Iniciação Científica e Mestrado.

\section{Referências}

Alencar, A. P. A., de Matos, J. H. F., de Souza, J. F., Marques, V. M. C., Lira, P. F., Moreira, A. E. A., \& da Silva Laurentino, P. A. (2020). Itinerário terapêutico de mulheres com câncer. Brazilian Journal of Development, 6(6), 42023-42035. 
Almeida, T. G. D., Comassetto, I., Alves, K. D. M. C., Santos, A. A. P. D., Silva, J. M. D. O., \& Trezza, M. C. S. F. (2015). Vivência da mulher jovem com câncer de mama e mastectomizada. Escola Anna Nery, 19, 432-438.

Andrade, A. F. S. M., de Santana Teles, W., da Silva, M. C., Torres, R. C., Debbo, A., Azevedo, M. V. C., ... \& Calasans, T. A. S. (2021). Mulher mastectomizada e repercussões em seu cotidiano: verificação supletiva. Research, Society and Development, 10(11).

Bardin L. (2011). Análise de conteúdo. SP: Edições 70.

Conde, C. R., Lemos, T. M. R., Pozati, M. P. S., \& Marques, M. D. L. D. S. (2016). A repercussão do diagnóstico e tratamento do câncer de mama no contexto familiar. Revista Uningá, 47(1).

Costa, A. P., Linhares, R., \& Souza, F. D. (2012). Possibilidades de Análise Qualitativa no webQDA e colaboração entre pesquisadores em educação em comunicação. Anais $3^{\circ}$ Simpósio Educação e Comunicação: Infoinclusão possibilidades de ensinar e aprender, $276-286$.

Costa, D. T., Silva, D. M. R. D., Cavalcanti, I. D. L., Gomes, E. T., Vasconcelos, J. L. D. A., \& Carvalho, M. V. G. D. (2019). Religious/spiritual coping and level of hope in patients with cancer in chemotherapy. Revista brasileira de enfermagem, 72, 640-645.

Bitencourt, J. F. V., \& de Oliveira Souza, I. E. (2015). Necessidades de mulheres no enfrentamento do diagnóstico de câncer de mama e do tratamento cirúrgico. Revista de Enfermagem da UFJF, 1(2).

Dias, E. N., \& Pais-Ribeiro, J. L. (2019). O modelo de coping de Folkman e Lazarus: aspectos históricos e conceituais. Revista Psicologia e Saúde, 11(2), 5566.

Fernandes, V. (2016). O impacto do câncer de mama e a importância da atuação da equipe de enfermagem. <http://www.enfermeiroaprendiz.com.br/oimpacto-do-cancer-de-mama-e-importancia-da-atuacao-da-equipe-de-enfermagem/>.

Fonseca, A. B. C., Rodrigues, E. S. R. C., Nobrega, M. M., Nobre, J. O. C., França, G. J., \& Silva, L. P. (2016). Estimativa para o câncer de mama feminino: e a assistência de enfermagem na prevenção. Rev. Temas e Saúde, 16(4), 14-30.

Gonzalez, P., Nuñez, A., Wang-Letzkus, M., Lim, J. W., Flores, K. F., \& Nápoles, A. M. (2016). Coping with breast cancer: Reflections from Chinese American, Korean American, and Mexican American women. Health Psychology, 35(1), 19.

Herdman, T. H. (2018). NANDA-I Diagnosis Keperawatan Definisi dan Klasifikasi 2018-2020.

Instituto Nacional do Câncer (BR). (2021). Controle do Câncer de Mama. <https://www.inca.gov.br/mama>

Lacerda, C. S., Balbino, C. M., Sá, S. P. C., Silvino, Z. R., da Silva Júnior, P. F., Gomes, E. D. N. F., \& Joaquim, F. L. (2020). Enfrentamento de mulheres com câncer de mama. Research, Society and Development, 9(7), e165974018-e165974018.

Machado, M. X., Soares, D. A., \& Oliveira, S. B. (2017). Significados do câncer de mama para mulheres no contexto do tratamento quimioterápico. Physis: Revista de Saúde Coletiva, 27, 433-451.

Migowski, A. Silva, G.A.S. Dias, M.B.K. Diz, M.D.P.E. Sant'ana, D.R. Nadanowski, P. Diretrizes para detecção precoce do câncer de mama no Brasil. II Novas recomendações nacionais, principais evidências e controvérsias. Cad. Saúde Pública; 34(6):e00074817, 2018.

Minayo, M.C.S. (2013) O desafio do conhecimento: pesquisa qualitativa em saúde.Revista e ampliada. São Paulo: Hucitec.

Paganini, J. (2016). Lei garante reconstrução da mama em seguida à retirada de câncer. Recuperado em 22 de março de 2020 , <http://www12.senado.leg.br/noticias/materias/2016/05/07/lei-garante-reconstrucao-da-mama-em-seguida-a-retirada-de-cancer>.

Paiva, A. D. C. P. C., Elias, E. A., Souza, Í. E. D. O., Moreira, M. C., Melo, M. C. S. C. D., \& Amorim, T. V. (2020). Cuidado de enfermagem na perspectiva do mundo da vida da mulher-que-vivencia-linfedema-decorrente-do-tratamento-de-câncer-de-mama. Escola Anna Nery, 24.

Pereira, A.S. et al. (2018). Metodologia da pesquisa científica. [e-book]. Santa Maria. Ed. UAB/NTE/UFSM.

Pontes, B. F., Quitete, J. B., dos Reis, R. F., da Silva, B. M. S., da Silva, M. T., da Silveira, S. S. D., \& de Carvalho Castro, R. (2020). Outubro rosa: uma ação de cuidado no cenário das políticas públicas. Brazilian Journal of Development, 6(6), 34504-34518.

Reis, A. P. A., Panobianco, M. S., \& Gradim, C. V. C. (2019). Enfrentamento de mulheres que vivenciaram o câncer de mama. Revista de Enfermagem do Centro-Oeste Mineiro, 9.

Sousa, F. F. D. P. R. D., Freitas, S. M. F. D. M., Farias, A. G. D. S., Cunha, M. D. C. D. S. O., Araújo, M. F. M. D., \& Veras, V. S. (2017). Religious/spiritual coping by people with cancer undergoing chemotherapy: integrative literature review. SMAD. Revista eletrônica saúde mental álcool e drogas, 13(1), 45-51.

Souza, V. R. D. S., Marziale, M. H. P., Silva, G. T. R., \& Nascimento, P. L. (2021). Tradução e validação para a língua portuguesa e avaliação do guia COREQ. Acta Paulista de Enfermagem, 34.

Yoshinari, S. T. V., Júnior, G. H. Y., Masson, M. V., \& de Mello, L. F. (2017). Vivência de mulheres frente ao câncer de mama: revisão da literatura brasileira/The experience of women facing breast cancer: a review of Brazilian scientific literature. Health Sciences Journal, 7(4), $20-25$. 